\title{
The M4 adaptive unit for the E-ELT
}

B Crépy (b), S Chaillot (g), JM Conan (d), R Cousty (b), C Delrez (c), M Dimmler (a), JL Dournaux (f), S De Zotti (e), E Gabriel (c), R Gasmi (f), R Grasser (b), N Hubin (a), P Jagourel (f), L Jochum (a), F Locre (b), P-Y Madec (a), P Morin (b), M Mueller (a), G Petit (d), D Petitgas (b), JJ Roland (b), JC Sinquin (b), E Vernet (a)

ESO (a) Karl-Schwarzschild-Strasse 2 - D-85748 Garching bei München CILAS (b) , 8 Avenue Buffon - BP 6319 - 45063 Orléans Cedex, France AMOS (C Liege science park - Rue des Chasseurs Ardennais - 4031 Angleur, Belgium ONERA (d) BP72 - 29 avenue de la Division Leclerc - FR-92322 - Chatillon, France Astrium (e), 31, avenue des Cosmonautes - 31402 Toulouse, France Observatory of Paris Meudon (f), 5, place Jules Janssen, 92195 Meudon, France Boostec (g), Zone Industrielle - 65460 Bazet, France

\begin{abstract}
Cilas proposes a M4 adaptive mirror (M4AM) that corrects the atmospheric turbulence at high frequencies and residual tip-tilt and defocus due to telescope vibrations by using piezostack actuators. The design presents a matrix of nearly 7000 actuators (hexagonal geometry, spacing equal to $29 \mathrm{~mm}$ ) leading to a fitting error simulated by Onera reaching the fitting error goal. The mirror is held by a positioning system which ensures all movements of the mirror at low frequency and selects the focus (Nasmyth A or B) using a hexapod concept. This subsystem is fixed rigidly to the mounting system and permits mirror displacements. The M4 control system (M4CS) ensures the connection between the telescope control/monitoring system and the M4 unit - positioning system (M4PS) and piezostack actuators in particular. This subsystem is composed of electronic boards, mechanical support fixed to the mounting structure and the thermal hardware. With piezostack actuators, most of the thermal load is minimized and dissipated in the electronic boards and not in the adaptive mirror. The mounting structure (M4MS) is the mechanical interface with the telescope (and the ARU in particular) and ensures the integrity and stability of M4 unit subsystems. M4 positioning system and mounting structure are subcontracted to Amos company. We will also report on the manufacturing of the demonstration prototype that will be tested in the next phase.
\end{abstract}

Keywords: Adaptive optic, Adaptive unit, E-ELT, hexapod, mirror, PZT actuator

\section{INTRODUCTION}

ESO has initiated in October 2007 a preliminary study to demonstrate the feasibility of the M4AU, adaptive unit of $\phi 2,6 \mathrm{~m}$, for the European extremely large telescope (E-ELT).

CILAS has proposed a concept based on piezostack technology that fulfils all the requirements (no show stopper identified). Breadboards and demonstration prototype are manufactured and tested to validate our present assumptions and to mitigate the risks.

\section{ANALYSIS OF THE MAIN REQUIREMENTS}

The M4AU is designed in collaboration between CILAS (Orleans in France) and AMOS (Liege in Belgium). It consists of four main units: The M4AM (adaptive mirror), the M4CS (control system) designed by CILAS and the M4PS (positioning system) and M4MS (mounting structure) designed by AMOS.

The main function of the M4 adaptive unit is to correct the aberrations of the wave front due to the following perturbations:

Atmospheric turbulences

Telescope vibrations and deformations

á Wind

This is an Open Access article distributed under the terms of the Creative Commons Attribution-Noncommercial License, which permits unrestricted use, distribution, and reproduction in any noncommercial medium, provided the original work is properly cited. 
The M4AM corrects the atmospheric turbulences at high frequencies by using piezo electric actuators. This subsystem is fixed to the M4PS.

The M4PS ensures all movements of the M4AM at low frequency and select the focus (Nasmyth A or B). It corrects essentially the telescope vibrations, the telescope deformations and the wind. This subsystem is fixed rigidly to the M4MS and permits M4AM displacements. The different kinds of displacements are:

a Translation movements in plane $\mathrm{X}, \mathrm{Y}$

a Swinging movements around $X$ and $Y$

a Rotation around $\mathrm{Y}$ to address the Nasmyth foci

The M4CS ensures the connection between the telescope control / monitoring system and the rest of the M4AU (the adaptive mirror and the positioning system in particular). This subsystem is composed of electronic boards, mechanical support fixed to the M4MS and the thermal hardware. With piezo actuators, most of the thermal load is minimized and dissipated in the electronic boards and not in the adaptive mirror. The thermal architecture is simplified.

The M4MS is the mechanical interface with the telescope (and the ARU in particular) and ensures the integrity and stability of M4AU subsystems.

We not intend here to summarize all the requirements but to explicit those that impact the overall architecture:

a Mirror outer diameter is $2,6 \mathrm{~m}$ and inner diameter is $0,7 \mathrm{~m}$, slightly elliptical $(2 \%)$. Regarding the slight ellipticity, the mirror will be circular and will be the largest adaptive mirror ever built.

* The fitting error shall be less than $145 \mathrm{~nm}$ leading to a very high quantity of actuators. The analysis performed by Onera showed that around 7217 actuators are necessary in a triangular meshing to reach the fitting error goal of $110 \mathrm{~nm}$.

a Correction capability of the mirror (see analysis in next paragraph). The desired stroke is proportional to the length of the actuators and directly linked to the thickness of the mirror.

a First eigen frequency shall be higher than $20 \mathrm{~Hz}$. As a consequence, the structure shall be very stiff.

a Mass shall be lower than 10 tons involving the use of lightweight parts such as the mirror base plate made of silicone carbide.

Local flatness shall be less than $15 \mathrm{~nm}$. No print through are admitted. The interface between the actuator and the optical plate shall be designed carefully. Nevertheless, the type of performances was achieved with previous design made by CILAS.

a Global flatness shall be lower than $250 \mathrm{~nm}$. In open loop, this requirement is easily reached by the stroke correction provided by the PZT actuators. In open loop, we shall minimize the impact of the creep and the hysteresis of the PZT actuators. A breadboard is dedicated to the demonstration of these performances.

a The temperature of all external surfaces of the M4AU including the surface of the mirror itself, in the absence of wind shall not differ from that of the ambient air by more than $\pm 1^{\circ} \mathrm{C}$. Our thermal architecture is based on the following assumptions: Passive thermal control of the external covers (low inertia, thermally decoupling with the housing structures), exchanges with the sky limited $\left(-50^{\circ} \mathrm{C}\right)$, no internal dissipation in the M4AM, cooling fluid reserved to M4CS dissipation since no internal dissipation in the M4AM.

* Cross coupling of the positioning system shall be less than 0,25 arcsec and less than 0,025 arcsec during any time period of $0,2 \mathrm{~s}$. This requirement is challenging for the hexapod and greatly impacts the quality of the mechanical pieces of the hexapod but also the strategy of command of this sub system.

aTBF better than 3 years, lifetime 30 years leads to use reliable technologies and to develop specific maintenance concepts (including preventive ones)

\section{OVERVIEW OF THE PROPOSED DESIGN}

The M4 adaptive unit is part of the Adaptive Relay Unit (ARU) tower of the E-ELT (see figure 1). The optical beam coming from the M2 cross the mirror in its centre and is reflected by the M3, located bellow the M4. The M4AU shall then reflect the optical beam from M3 to M5. The M4AU is attached to the ARU tower in 6 points. A dedicated tool (not shown) is designed in order to transport the unit to the load area close to the telescope, to support and interface the unit with the crane integrated in the telescope and to be compatible with the integration procedure of the unit in the tower.

The conceptual design of the M4AU is presented in figure 2. The next paragraphs give the s a brief description of the four sub systems of the M4AU. 


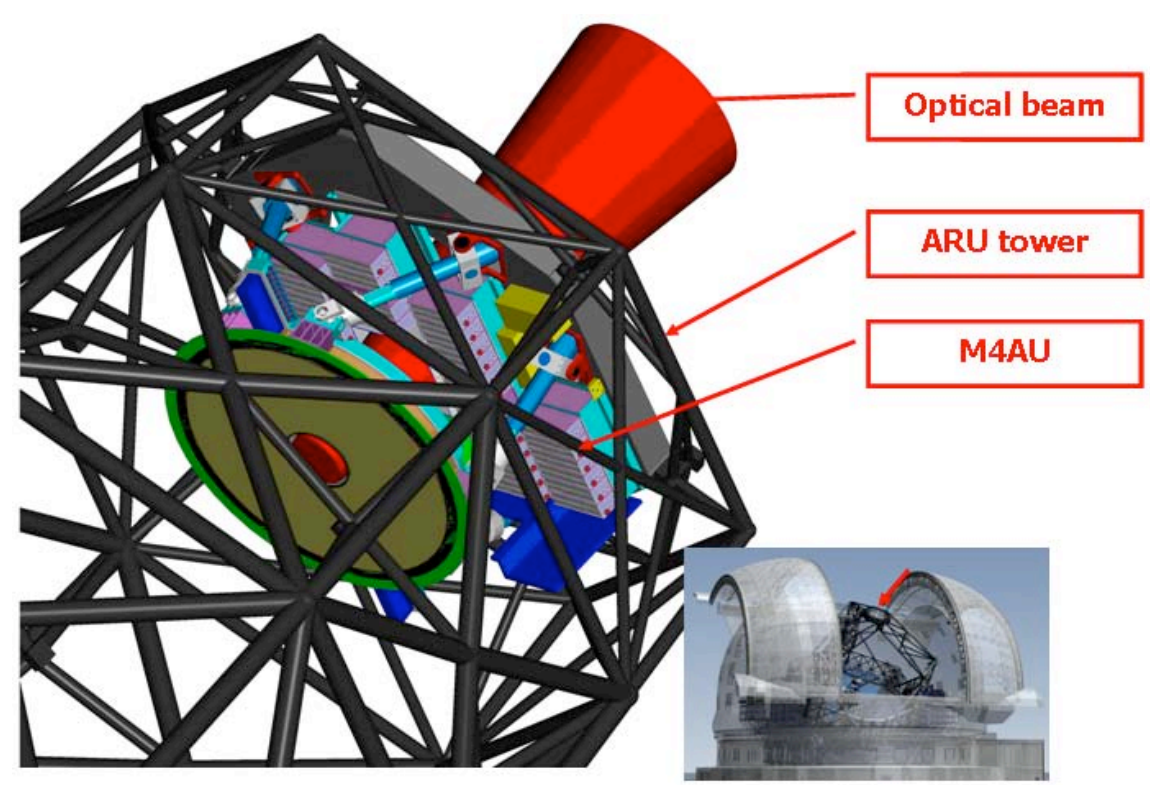

figure 1: The M4 adaptive unit fixed on the adaptive relay unit (ARU) tower of the E-ELT

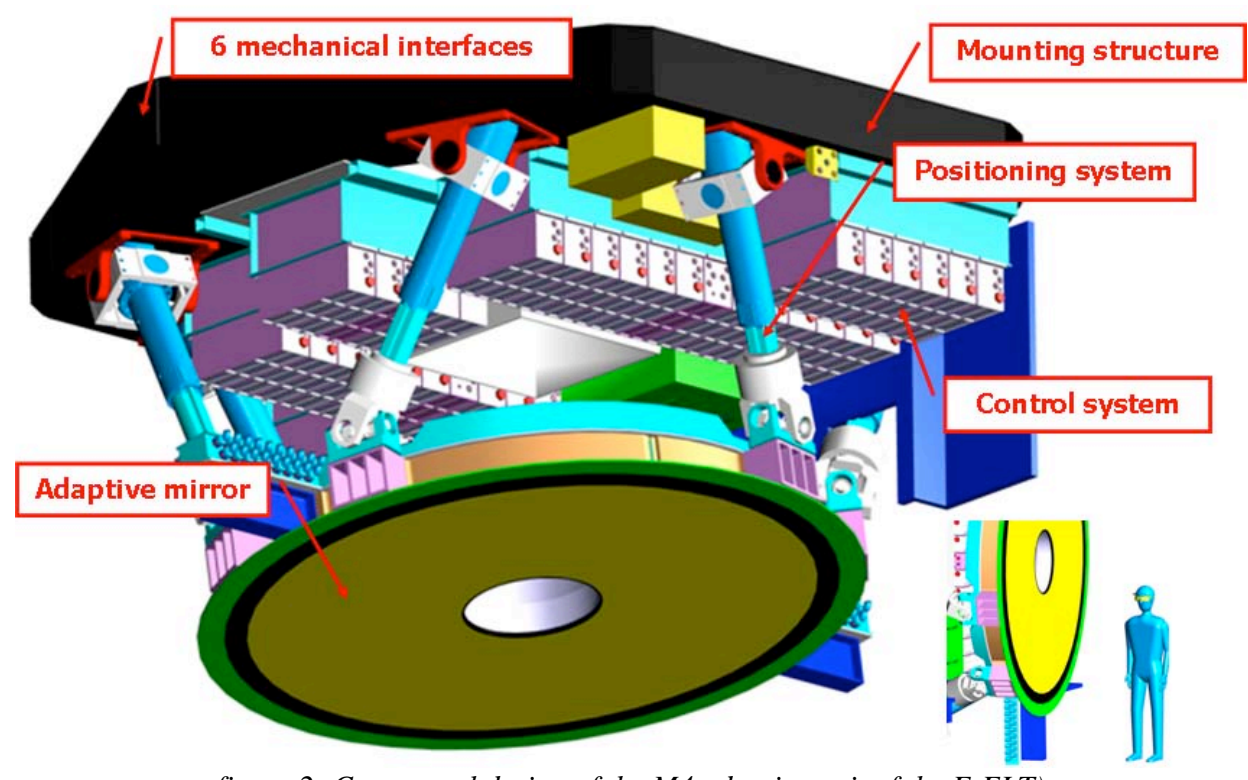

figure 2: Conceptual design of the M4 adaptive unit of the E-ELT)

The deformable mirror (M4AM) is mainly composed of the following assemblies:

a The optical plate made in Zerodur ${ }^{\circledR}$ has a thickness of $4 \mathrm{~mm}$ and is rigidly mounted on the PZT actuators. This optical plate is deformed by the PZT actuators.

* The actuators are arranged in a triangular geometry and based upon a piezoelectric technology which is usual for CILAS deformable mirrors (SAM=Stacked Array Mirror). 6933 actuators are integrated the clear aperture leading to an inter actuator distance of $29 \mathrm{~mm}$.

a The structural base plate is made of silicone carbide and has a thickness of $250 \mathrm{~mm}$. The main function of this base plate is to provide stiffness to the mirror.

a The metallic base plate made in INVAR is attached to the structural base plate and permit the accurate fixing of the actuators at the back side of the mirror (see figure 3 ).

* High voltage distribution PCBs close to the actuators simplify the electrical connection and provide access to the actuators if exchange is required

a Main electrical connectors are mounted on a metallic ring and are located along the Nasmyth rotation axis to avoid stress in the cables during each focus selection 
a Covers (central, front and rear) protect the mirror from dust and humidity.

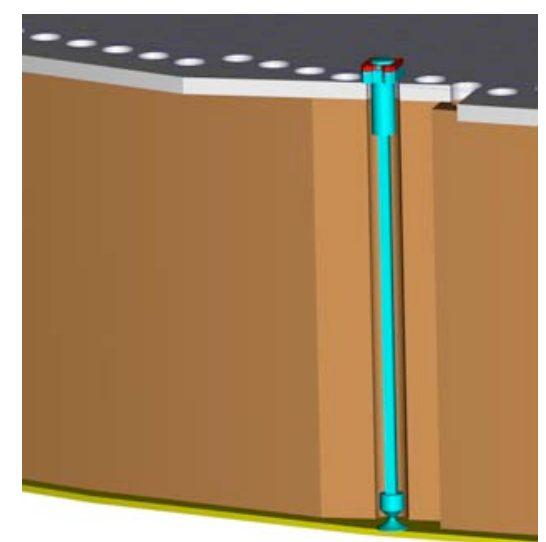

figure 3: Cut view of the M4 adaptive mirror showing the integration of the PZT actuator in the mirror

The stroke need for the mirror is the following:

- Static stroke : $25 \mu \mathrm{m}$

á Dynamical stroke : $50 \mu \mathrm{m}$

Summing these two contributions, we obtain a requirement for the total stroke of $75 \mu \mathrm{m}$. Our present design allows a stroke capability of $80 \mu \mathrm{m}$.

The following figure shows the typical influence function (stroke up to $10 \mu \mathrm{m}$ ) that corresponds to the maximum voltage applied to one actuator and the inter actuator stroke value of $4,3 \mu \mathrm{m}$.

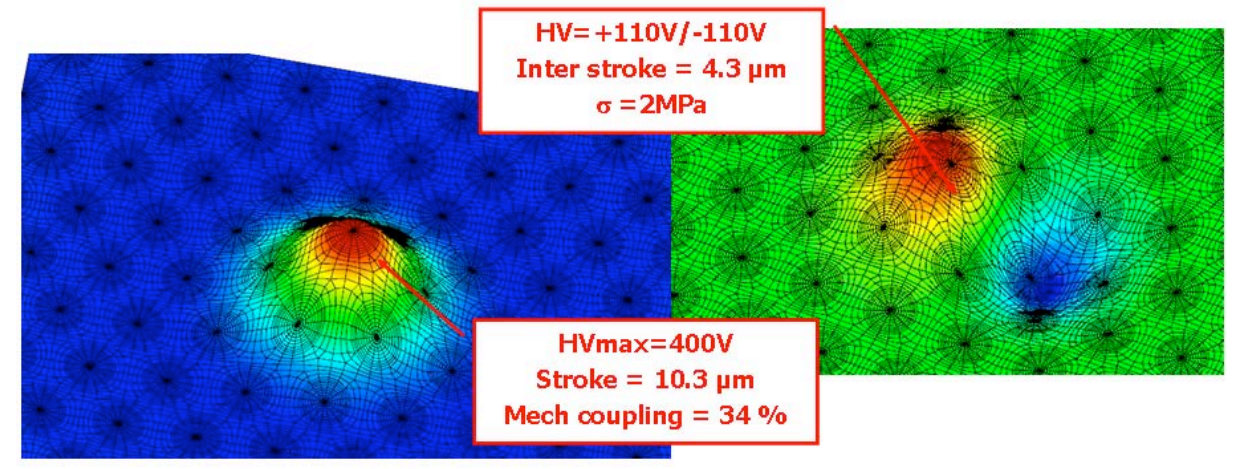

figure 4: Influence function (left) and inter actuator stroke of the M4 adaptive mirror (right)

The positioning system (M4PS) is a hexapod. The six feet holding the mirror are fixed on the mounting structure (see figure 2). The hexapod allows the M4 positioning and switching between focal stations. A foot is composed of one actuator, one cardan and one ball joint (made with one cardan and one axial bearing).

\begin{tabular}{|l|c|}
\hline \multicolumn{1}{|c|}{ Parameter } & Value \\
\hline Stroke per foot & $400 \mathrm{~mm}$ \\
\hline Resolution per actuator & $2 \mu \mathrm{m}$ \\
\hline Mass & $543 \mathrm{~kg} \mathrm{x6}$ \\
\hline Stiffness per actuator & $360 \mathrm{~N} / \mu \mathrm{m}$ \\
\hline Max cross coupling & $0,21 \mathrm{arcsec} \mathrm{PV}$ (max) \\
\hline
\end{tabular}

table 1: Main parameters of the M4 positioning system

The M4MS (mounting structure) is made of shell elements. The main structure made of steel presents optimized shell thicknesses for a total mass near one ton.

The M4CS (control system) is the electronic part of the M4AU. This electronic drives the M4PS and the M4AM piezo actuators with high voltages (in the $+/-400 \mathrm{~V}$ range). All the actions made by the M4CS in order to drive the M4AM and the M4PS are due to external orders received by the M4CS.

The M4CS is also in charge of the housekeeping of the M4AU. 
The figure below shows the functional architecture of the M4AU.

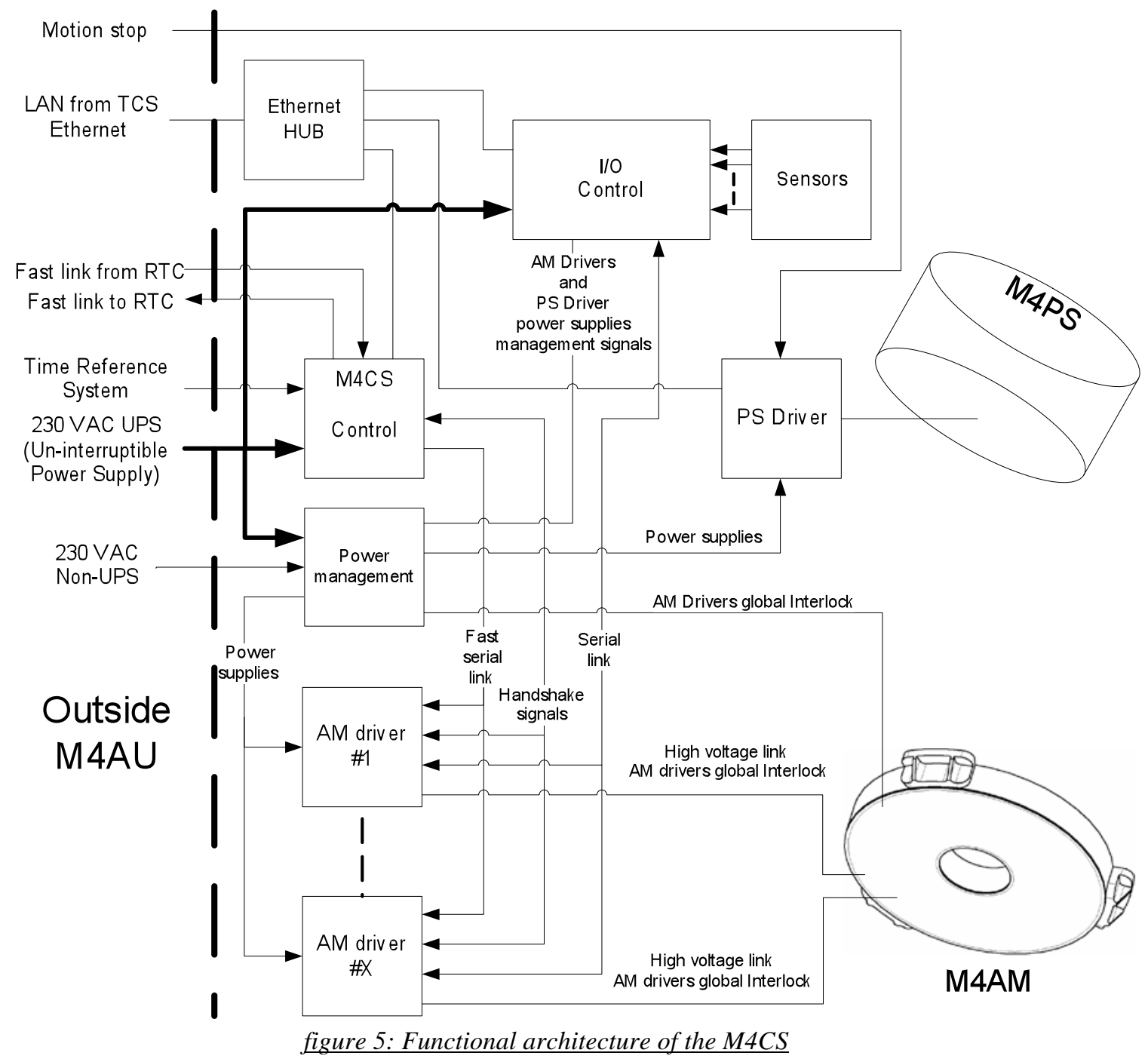

The main equipments of the M4CS are the AM Drivers, the M4CS Control Unit, the I/O Control Unit and the PS Driver cabinet.

The AM Driver generates the high voltage applied to the actuators. These high voltages are generated from the commands send by the M4CS Control. The AM Driver checks the good working order of each high voltage amplifier channel. Moreover, it can test each actuator line continuity and actuator capacitor during continuous operation.

The M4CS Control is in charge of the data exchange with the TCS and the RTC and the main internal sub systems, the computation of actuator commands and tilts command, the change of mode and the control of the modes and the housekeeping of the M4AU.

The I/O Control Unit performs the control of the AM Drivers according M4CS Control orders, collect of the signals coming from the sensors and manage the power supplies of the AM Drivers and of the PS Driver.

The M4PS is controlled by the M4CS PS Control.

\section{DESCRIPTION OF THE DEMONSTRATION PROTOTYPE}


Adaptive optical mirror with diameter of more than $500 \mathrm{~mm}$ are unusual for CILAS however we are confident our technology is scalable. In order to address the specific issues relative to large adaptive mirrors, CILAS had proposed to design, manufacture and test a demonstration prototype with the following main characteristics:

Total number of actuators: 852

a Meshing geometry : Square

Inter actuator distance: $24,5 \mathrm{~mm}$

a Optical plate diameter $=871 \mathrm{~mm}$

a Mass $=230 \mathrm{~kg}$

The main objectives of this demonstration prototype are:

* To experimentally study the global dynamical behaviour

a To experimentally study the global thermal behaviour

To demonstrate the manufacturing process and to learn from this experience for the future $2.6 \mathrm{~m}$ mirror

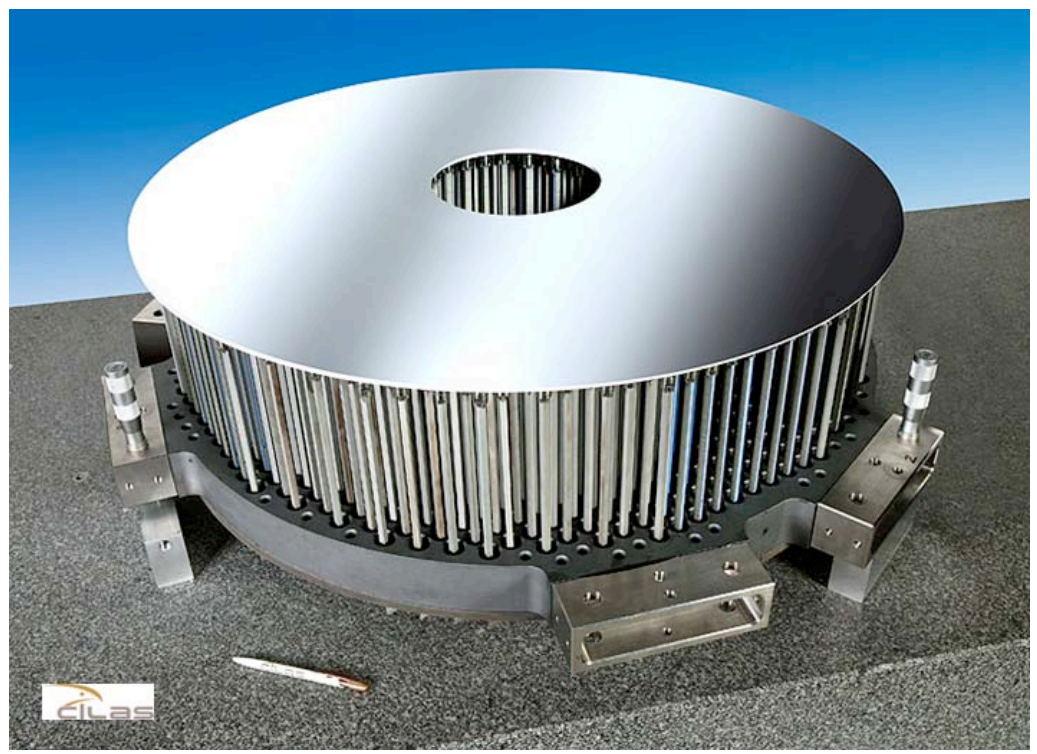

figure 6: Demonstration prototype of the M4 adaptive mirror manufactured in less than 1 year

\section{CONCLUSIONS}

CILAS has successfully passed the conceptual design review and is beginning the test of the demonstration prototype in parallel with the preliminary design of the M4AU.

\section{ACKNOWLEDGEMENTS}

This work has been made in a framework of a contract with the European Organisation for Astronomical Research in the Southern Hemisphere (ESO)

\section{REFERENCES}

[1] L. Jochum et al, Pre-focal wave front correction and field stabilization for the E-ELT, in this conference;

[2] JC. Sinquin et al, Recent Progress on Correcting Components at CILAS, in this conference; 